\title{
Factors of alternating sums of products of binomial and $q$-binomial coefficients
}

\author{
by \\ ViCTOR J. W. Guo (Shanghai), FrÉDÉRIC Jouhet (Lyon) \\ and JiANG ZENG (Lyon)
}

1. Introduction. In 1998, Calkin [4] proved that, for all positive integers $m$ and $n$,

$$
\left(\begin{array}{c}
2 n \\
n
\end{array}\right)^{-1} \sum_{k=-n}^{n}(-1)^{k}\left(\begin{array}{c}
2 n \\
n+k
\end{array}\right)^{m}
$$

is an integer by arithmetical techniques. For $m=1,2$ and 3 , by the binomial theorem, Kummer's formula and Dixon's formula, it is easy to see that (1.1) is equal to 0,1 and $\left(\begin{array}{c}3 n \\ n\end{array}\right)$, respectively. Recently in the study of finite forms of the Rogers-Ramanujan identities [7] we stumbled across (1.1) for $m=4$ and $m=5$, which gives

$$
\sum_{k=0}^{n}\left(\begin{array}{c}
2 n+k \\
k
\end{array}\right)\left(\begin{array}{c}
2 n \\
n+k
\end{array}\right)^{2} \text { and } \sum_{k=0}^{n}\left(\begin{array}{c}
3 n-k \\
n-k
\end{array}\right)\left(\begin{array}{c}
2 n+k \\
k
\end{array}\right)\left(\begin{array}{c}
2 n \\
n+k
\end{array}\right)^{2},
$$

respectively. Indeed, de Bruijn [3] has shown that for $m \geq 4$ there is no closed form for (1.1) by asymptotic techniques. Our first objective is to give a $q$-analogue of Calkin's result, which also implies that (1.1) is positive for $m \geq 2$.

In 2004, Zudilin [14] proved that for all positive integers $n, j$ and $r$,

$$
\left(\begin{array}{c}
2 n \\
n
\end{array}\right)^{-1}\left(\begin{array}{c}
2 j \\
j
\end{array}\right) \sum_{k=j}^{n}(-1)^{n-k} \frac{2 k+1}{n+k+1}\left(\begin{array}{c}
2 n \\
n-k
\end{array}\right)\left(\begin{array}{l}
k+j \\
k-j
\end{array}\right)^{r} \in \mathbb{Z},
$$

which was originally observed by Strehl [12] in 1994. In fact, Zudilin's motivation was to solve the following problem, which was raised by Schmidt [11] in 1993 and was apparently not related to Calkin's result.

2000 Mathematics Subject Classification: 05A10, 05A30, $11 \mathrm{~B} 65$. 
Problem 1.1 (Schmidt [11]). For any integer $r \geq 2$, define a sequence of numbers $\left\{c_{k}^{(r)}\right\}_{k \in \mathbb{N}}$, independent of the parameter $n$, by

$$
\sum_{k=0}^{n}\left(\begin{array}{l}
n \\
k
\end{array}\right)^{r}\left(\begin{array}{c}
n+k \\
k
\end{array}\right)^{r}=\sum_{k=0}^{n}\left(\begin{array}{l}
n \\
k
\end{array}\right)\left(\begin{array}{c}
n+k \\
k
\end{array}\right) c_{k}^{(r)} .
$$

Is it true that all the numbers $c_{k}^{(r)}$ are integers?

At the end of his paper, Zudilin [14] raised the problem of finding and solving a $q$-analogue of Problem 1.1. Our second objective is to provide such a $q$-analogue.

For any integer $n$, define the $q$-shifted factorial $(a)_{n}$ by $(a)_{0}=1$ and

$$
(a)_{n}= \begin{cases}(1-a)(1-a q) \cdots\left(1-a q^{n-1}\right), & n=1,2, \ldots, \\ \left(\left(1-a q^{-1}\right)\left(1-a q^{-2}\right) \cdots\left(1-a q^{n}\right)\right)^{-1}, & n=-1,-2, \ldots\end{cases}
$$

We will also use the compact notations for $m \geq 1$ :

$$
\left(a_{1}, \ldots, a_{m}\right)_{n}:=\left(a_{1}\right)_{n} \cdots\left(a_{m}\right)_{n}, \quad\left(a_{1}, \ldots, a_{m}\right)_{\infty}:=\lim _{n \rightarrow \infty}\left(a_{1}, \ldots, a_{m}\right)_{n} .
$$

The $q$-binomial coefficients are defined as

$$
\left[\begin{array}{l}
n \\
k
\end{array}\right]:=\left[\begin{array}{l}
n \\
k
\end{array}\right]_{q}=\frac{(q)_{n}}{(q)_{k}(q)_{n-k}} .
$$

Since $1 /(q)_{n}=0$ if $n<0$, we have $\left[\begin{array}{l}n \\ k\end{array}\right]=0$ if $k>n$ or $k<0$.

The following is our first generalization of Calkin's result.

Theorem 1.2. For $m \geq 3$ and all positive integers $n_{1}, \ldots, n_{m}$,

$$
\begin{aligned}
\sum_{k=-n_{1}}^{n_{1}}(-1)^{k} q^{(m-1)} k^{2}+\left(\begin{array}{l}
k \\
2
\end{array}\right) \prod_{i=1}^{m}\left[\begin{array}{c}
n_{i}+n_{i+1} \\
n_{i}+k
\end{array}\right] \\
=\left[\begin{array}{c}
n_{1}+n_{m} \\
n_{1}
\end{array}\right] \sum_{\lambda} \prod_{i=1}^{m-2} q^{\lambda_{i}^{2}}\left[\begin{array}{c}
\lambda_{i-1} \\
\lambda_{i}
\end{array}\right]\left[\begin{array}{c}
n_{i+1}+n_{i+2} \\
n_{i+1}-\lambda_{i}
\end{array}\right],
\end{aligned}
$$

where $n_{m+1}=\lambda_{0}=n_{1}$ and the sum is over all sequences $\lambda=\left(\lambda_{1}, \ldots, \lambda_{m-2}\right)$ of nonnegative integers such that $\lambda_{0} \geq \lambda_{1} \geq \cdots \geq \lambda_{m-2}$.

Calkin [4] has given a partial $q$-analogue of (1.1) by considering the alternating sum $\sum_{k=0}^{n}(-1)^{k} q^{j k}\left[\begin{array}{c}n \\ k\end{array}\right]^{m}$. In this respect, besides (1.3), we shall also prove the following divisibility result.

Theorem 1.3. For all positive integers $n_{1}, \ldots, n_{m}, n_{m+1}=n_{1}$, the alternating sum

$$
S\left(n_{1}, \ldots, n_{m} ; j, q\right):=\left[\begin{array}{c}
n_{1}+n_{m} \\
n_{1}
\end{array}\right]^{-1} \sum_{k=-n_{1}}^{n_{1}}(-1)^{k} q^{j k^{2}+\left(\begin{array}{c}
k \\
2
\end{array}\right)} \prod_{i=1}^{m}\left[\begin{array}{c}
n_{i}+n_{i+1} \\
n_{i}+k
\end{array}\right]
$$

is a polynomial in $q$ with nonnegative integral coefficients for $0 \leq j \leq m-1$. 
We shall give two proofs of Theorem 1.2. The first one is based on a recurrence relation formula for $S\left(n_{1}, \ldots, n_{m} ; j, q\right)$, which also leads to a proof of Theorem 1.3. The second one follows directly from Andrews' basic hypergeometric identity between a single sum and a multiple sum [1, Theorem 4]:

Theorem 1.4 (Andrews [1]). For every integer $m \geq 0$, the following identity holds:

$$
\begin{aligned}
& \sum_{k \geq 0} \frac{\left(a, q \sqrt{a},-q \sqrt{a}, b_{1}, c_{1}, \ldots, b_{m}, c_{m}, q^{-N}\right)_{k}}{\left(q, \sqrt{a},-\sqrt{a}, a q / b_{1}, a q / c_{1}, \ldots, a q / b_{m}, a q / c_{m}, a q^{N+1}\right)_{k}} \\
& \times\left(\frac{a^{m} q^{m+N}}{b_{1} c_{1} \cdots b_{m} c_{m}}\right)^{k} \\
& =\frac{\left(a q, a q / b_{m} c_{m}\right)_{N}}{\left(a q / b_{m}, a q / c_{m}\right)_{N}} \sum_{l_{1}, \ldots, l_{m-1} \geq 0} \frac{\left(a q / b_{1} c_{1}\right)_{l_{1}} \cdots\left(a q / b_{m-1} c_{m-1}\right)_{l_{m-1}}}{(q)_{l_{1}} \cdots(q)_{l_{m-1}}} \\
& \times \frac{\left(b_{2}, c_{2}\right)_{l_{1}} \cdots\left(b_{m}, c_{m}\right){ }_{l_{1}+\cdots+l_{m-1}}}{\left(a q / b_{1}, a q / c_{1}\right)_{l_{1}} \cdots\left(a q / b_{m-1}, a q / c_{m-1}\right)_{l_{1}+\cdots+l_{m-1}}} \\
& \times \frac{\left(q^{-N}\right)_{l_{1}+\cdots+l_{m-1}}}{\left(b_{m} c_{m} q^{-N} / a\right)_{l_{1}+\cdots+l_{m-1}}} \frac{(a q)^{l_{m-2}+\cdots+(m-2) l_{1}} q^{l_{1}+\cdots+l_{m-1}}}{\left(b_{2} c_{2}\right)^{l_{1}} \cdots\left(b_{m-1} c_{m-1}\right)^{l_{1}+\cdots+l_{m-2}}} .
\end{aligned}
$$

Note that there are two ingredients in Zudilin's approach to Problem 1.1: one is Theorem 1.4 with $q=1$ and the other is the Legendre transform. Now, a $q$-Legendre transform reads:

$$
a_{n}=\sum_{k=0}^{n} q^{\left(\begin{array}{c}
n-k \\
2
\end{array}\right)}\left[\begin{array}{l}
n+k \\
n-k
\end{array}\right] b_{k} \Leftrightarrow b_{n}=\sum_{k=0}^{n}(-1)^{n-k} \frac{1-q^{2 k+1}}{1-q^{n+k+1}}\left[\begin{array}{c}
2 n \\
n-k
\end{array}\right] a_{k}
$$

which is a special case of Carlitz's q-Gould-Hsu inverse formula [5] (see also [10]). Using (1.5) and Theorem 1.4 in its full generality we are able to formulate and prove a $q$-analogue of Problem 1.1.

THEOREM 1.5. For any integer $r \geq 1$, define rational fractions $c_{k}^{(r)}(q)$ of the variable $q$, independent of $n$, by writing

$$
\begin{aligned}
& \sum_{k=0}^{n} q^{r\left(\begin{array}{c}
n-k \\
2
\end{array}\right)+(1-r)\left(\begin{array}{l}
n \\
2
\end{array}\right)}\left[\begin{array}{l}
n \\
k
\end{array}\right]^{r}\left[\begin{array}{c}
n+k \\
k
\end{array}\right]^{r} \\
& =\sum_{k=0}^{n} q^{\left(\begin{array}{c}
n-k \\
2
\end{array}\right)+(1-r)\left(\begin{array}{l}
k \\
2
\end{array}\right)}\left[\begin{array}{l}
n \\
k
\end{array}\right]\left[\begin{array}{c}
n+k \\
k
\end{array}\right] c_{k}^{(r)}(q) .
\end{aligned}
$$

Then $c_{n}^{(r)}(q) \in \mathbb{N}[q]$.

REMARK. Since the $r=1$ case is trivial, we suppose that $r \geq 2$ in what follows. 

that

As $\left[\begin{array}{c}n \\ k\end{array}\right]\left[\begin{array}{c}n+k \\ k\end{array}\right]=\left[\begin{array}{c}2 k \\ k\end{array}\right]\left[\begin{array}{c}n+k \\ n-k\end{array}\right]$, invoking (1.5) we derive immediately from (1.6)

$$
q^{(1-r)\left(\begin{array}{c}
n \\
2
\end{array}\right)}\left[\begin{array}{c}
2 n \\
n
\end{array}\right] c_{n}^{(r)}(q)=\sum_{j=0}^{n}\left[\begin{array}{c}
2 j \\
j
\end{array}\right]^{r} t_{n, j}^{(r)}(q)
$$

where

$$
t_{n, j}^{(r)}(q)=q^{r\left(\begin{array}{c}
j+1 \\
2
\end{array}\right)} \sum_{k=j}^{n}(-1)^{n-k} \frac{1-q^{2 k+1}}{1-q^{n+k+1}}\left[\begin{array}{c}
2 n \\
n-k
\end{array}\right]\left[\begin{array}{c}
k+j \\
k-j
\end{array}\right]^{r} q^{\left(\begin{array}{c}
k \\
2
\end{array}\right)-r j k} .
$$

Therefore Theorem 1.5 is a consequence of the following theorem, which is our $q$-analogue of Zudilin's result (1.2).

TheOREM 1.6. For any integer $r \geq 2$,

$$
q^{(r-1)\left(\begin{array}{c}
n \\
2
\end{array}\right)}\left[\begin{array}{c}
2 j \\
j
\end{array}\right]\left[\begin{array}{c}
2 n \\
n
\end{array}\right]^{-1} t_{n, j}^{(r)}(q) \in \mathbb{N}[q] .
$$

As will be shown, Theorem 1.6 follows directly from Andrews' identity (1.4).

This paper is organized as follows. We prove Theorems 1.2 and 1.3 in the next section. The proof of Theorem 1.6 is given in Section 3. Some interesting divisibility results are given in Section 4. In the last section we present four related conjectures.

2. Proof of Theorems $\mathbf{1 . 2}$ and 1.3. We will need two known identities in $q$-series. One is the $q$-Pfaff-Saalschütz identity [6, Appendix, (II.12)] (see also $[8,13])$ :

$$
\begin{array}{r}
{\left[\begin{array}{c}
n_{1}+n_{2} \\
n_{1}+k
\end{array}\right]\left[\begin{array}{c}
n_{2}+n_{3} \\
n_{2}+k
\end{array}\right]\left[\begin{array}{c}
n_{3}+n_{1} \\
n_{3}+k
\end{array}\right]} \\
\quad=\sum_{r=0}^{n_{1}-k} \frac{q^{k^{2}+2 k r}(q)_{n_{1}+n_{2}+n_{3}-k-r}}{(q)_{r}(q)_{r+2 k}(q)_{n_{1}-k-r}(q)_{n_{2}-k-r}(q)_{n_{3}-k-r}}
\end{array}
$$

where $1 /(q)_{n}=0$ if $n<0$, and the other is the $q$-Dixon identity:

$$
\begin{array}{r}
\sum_{k=-n_{1}}^{n_{1}}(-1)^{k} q^{\left(3 k^{2}-k\right) / 2}\left[\begin{array}{c}
n_{1}+n_{2} \\
n_{1}+k
\end{array}\right]\left[\begin{array}{c}
n_{2}+n_{3} \\
n_{2}+k
\end{array}\right]\left[\begin{array}{c}
n_{3}+n_{1} \\
n_{3}+k
\end{array}\right] \\
=\frac{(q)_{n_{1}+n_{2}+n_{3}}}{(q)_{n_{1}}(q)_{n_{2}}(q)_{n_{3}}} .
\end{array}
$$

A short proof of (2.2) is given in [9].

We first establish the following recurrence formula. 
LEMma 2.1. Let $m \geq 3$. Then for all positive integers $n_{1}, \ldots, n_{m}$ and any integer $j$, the following recurrence holds:

$$
S\left(n_{1}, \ldots, n_{m} ; j, q\right)=\sum_{l=0}^{n_{1}} q^{l^{2}}\left[\begin{array}{c}
n_{1} \\
l
\end{array}\right]\left[\begin{array}{c}
n_{2}+n_{3} \\
n_{2}-l
\end{array}\right] S\left(l, n_{3}, \ldots, n_{m} ; j-1, q\right) .
$$

Proof. For any integer $k$ and positive integers $a_{1}, \ldots, a_{l}$, let

$$
C\left(a_{1}, \ldots, a_{l} ; k\right)=\prod_{i=1}^{l}\left[\begin{array}{c}
a_{i}+a_{i+1} \\
a_{i}+k
\end{array}\right],
$$

where $a_{l+1}=a_{1}$. Then

$$
S\left(n_{1}, \ldots, n_{m} ; j, q\right)=\frac{(q)_{n_{1}}(q)_{n_{m}}}{(q)_{n_{1}+n_{m}}} \sum_{k=-n_{1}}^{n_{1}}(-1)^{k} q^{j k^{2}+\left(\begin{array}{c}
k \\
2
\end{array}\right)} C\left(n_{1}, \ldots, n_{m} ; k\right) .
$$

We observe that for $m \geq 3$, we have

$$
C\left(n_{1}, \ldots, n_{m} ; k\right)=\frac{(q)_{n_{2}+n_{3}}(q)_{n_{m}+n_{1}}}{(q)_{n_{1}+n_{2}}(q)_{n_{m}+n_{3}}}\left[\begin{array}{c}
n_{1}+n_{2} \\
n_{1}+k
\end{array}\right]\left[\begin{array}{c}
n_{1}+n_{2} \\
n_{2}+k
\end{array}\right] C\left(n_{3}, \ldots, n_{m} ; k\right),
$$

and, by letting $n_{3} \rightarrow \infty$ in (2.1),

$$
\left[\begin{array}{c}
n_{1}+n_{2} \\
n_{1}+k
\end{array}\right]\left[\begin{array}{c}
n_{1}+n_{2} \\
n_{2}+k
\end{array}\right]=\sum_{r=0}^{n_{1}-k} \frac{q^{r^{2}+2 k r}(q)_{n_{1}+n_{2}}}{(q)_{r}(q)_{r+2 k}(q)_{n_{1}-k-r}(q)_{n_{2}-k-r}} .
$$

Plugging these into (2.4) we can write its right-hand side as

$$
R:=\sum_{k=-n_{1}}^{n_{1}} \sum_{r=0}^{n_{1}-k}(-1)^{k} C\left(n_{3}, \ldots, n_{m} ; k\right) \frac{q^{(r+k)^{2}+(j-1) k^{2}+\left(\begin{array}{c}
k \\
2
\end{array}\right)(q)_{n_{2}+n_{3}}(q)_{n_{1}}(q)_{n_{m}}}}{(q)_{r}(q)_{r+2 k}(q)_{n_{1}-k-r}(q)_{n_{2}-k-r}(q)_{n_{m}+n_{3}}} .
$$

Setting $l=r+k$, we see that $-n_{1} \leq l \leq n_{1}$, but if $l<0$, at least one of the indices $l+k$ and $l-k$ is negative for any integer $k$, which implies that $1 /(q)_{l-k}(q)_{l+k}=0$ by convention. Therefore, exchanging the order of summation, we have

$$
R=\sum_{l=0}^{n_{1}} \frac{q^{l^{2}}(q)_{n_{2}+n_{3}}(q)_{n_{1}}(q)_{n_{m}}}{(q)_{n_{1}-l}(q)_{n_{2}-l}(q)_{n_{m}+n_{3}}} \sum_{k=-l}^{l}(-1)^{k} C\left(n_{3}, \ldots, n_{m} ; k\right) \frac{q^{(j-1) k^{2}+\left(\begin{array}{l}
k \\
2
\end{array}\right)}}{(q)_{l-k}(q)_{l+k}} .
$$

Now, in the last sum making the substitution

$$
C\left(n_{3}, \ldots, n_{m} ; k\right)=\frac{(q)_{l-k}(q)_{l+k}(q)_{n_{m}+n_{3}}}{(q)_{n_{3}+l}(q)_{n_{m}+l}} C\left(l, n_{3}, \ldots, n_{m} ; k\right),
$$

we obtain the right-hand side of (2.3).

First proof of Theorem 1.2. Letting $n_{3} \rightarrow \infty$ in (2.2) yields

$$
S\left(n_{1}, n_{2} ; 1, q\right)=1 \text {. }
$$

Theorem 1.2 then follows by iterating $m-2$ times formula (2.3). 
Second proof of Theorem 1.2. Since

$$
\left[\begin{array}{c}
M \\
N+k
\end{array}\right]=(-1)^{k} q^{(M-N) k-\left(\begin{array}{c}
k \\
2
\end{array}\right)}\left[\begin{array}{c}
M \\
N
\end{array}\right] \frac{\left(q^{-M+N}\right)_{k}}{\left(q^{N+1}\right)_{k}},
$$

by collecting the terms of index $k$ and $-k$, the left-hand side of (1.3) can be written as

$$
\begin{aligned}
& L:=\prod_{i=1}^{m}\left[\begin{array}{c}
n_{i}+n_{i+1} \\
n_{i}
\end{array}\right]+\sum_{k=1}^{n_{1}}\left(1+q^{k}\right)(-1)^{k} q^{(m-1) k^{2}+\left(\begin{array}{c}
k \\
2
\end{array}\right)} \prod_{i=1}^{m}\left[\begin{array}{c}
n_{i}+n_{i+1} \\
n_{i}+k
\end{array}\right] \\
& =\prod_{i=1}^{m}\left[\begin{array}{c}
n_{i}+n_{i+1} \\
n_{i}
\end{array}\right]\left\{1+\sum_{k=1}^{n_{1}}\left(1+q^{k}\right)(-1)^{(m-1) k} q^{(m-1)\left(\begin{array}{c}
k+1 \\
2
\end{array}\right)} \prod_{i=1}^{m} q^{n_{i} k} \frac{\left(q^{-n_{i+1}}\right)_{k}}{\left(q^{n_{i}+1}\right)_{k}}\right\} .
\end{aligned}
$$

Letting $c_{1}=\cdots=c_{m}=c \rightarrow \infty$ and $a \rightarrow 1$ in Andrews' formula (1.4) we get

$$
\begin{aligned}
1+\sum_{k \geq 1}(1+ & \left.q^{k}\right) \frac{\left(b_{1}, \ldots, b_{m}, q^{-N}\right)_{k}}{\left(q / b_{1}, \ldots, q / b_{m}, q^{N+1}\right)_{k}}(-1)^{m k} q^{m\left(\begin{array}{c}
k \\
2
\end{array}\right)}\left(\frac{q^{m+N}}{b_{1} \cdots b_{m}}\right)^{k} \\
= & \frac{(q)_{N}}{\left(q / b_{m}\right)_{N}} \sum_{l_{1}, \ldots, l_{m-1} \geq 0} \frac{(q)_{N}}{(q)_{l_{1}} \cdots(q)_{l_{m-1}}(q)_{N-l_{1}-\cdots-l_{m-1}}} \\
& \times \prod_{i=1}^{m-1} \frac{\left(b_{i+1}\right)_{l_{1}+\cdots+l_{i}}}{\left(q / b_{i}\right)_{l_{1}+\cdots+l_{i}}}\left(\frac{-1}{b_{i+1}}\right)^{l_{1}+\cdots+l_{i}} q^{\left({ }_{l_{1}+\cdots+l_{i}}\right)+(m-i) l_{i}} .
\end{aligned}
$$

Now, shifting $m$ to $m-1$ in (2.6), setting

$$
N=n_{m}, \quad b_{i}=q^{-n_{i}} \quad \text { for } i=1, \ldots, m-1,
$$

and $\lambda_{i}=l_{1}+\cdots+l_{i}$ for $i=1, \ldots, m-2$, one sees that $L$ equals

$$
\begin{array}{r}
\prod_{i=1}^{m}\left[\begin{array}{c}
n_{i}+n_{i+1} \\
n_{i}
\end{array}\right] \frac{(q)_{n_{m}}^{2}}{\left(q^{1+n_{m-1}}\right)_{n_{m}}} \sum_{0 \leq \lambda_{1} \leq \cdots \leq \lambda_{m-2}} \prod_{i=1}^{m-2} \frac{\left(q^{-n_{i+1}}\right)_{\lambda_{i}}(-1)^{\lambda_{i}} q^{\left({ }^{\lambda_{i}+1}\right)+n_{i+1} \lambda_{i}}}{\left(q^{1+n_{i}}\right)_{\lambda_{i}}(q)_{\lambda_{i}-\lambda_{i-1}}} \\
=\left[\begin{array}{c}
n_{1}+n_{m} \\
n_{1}
\end{array}\right] \sum_{0 \leq \lambda_{1} \leq \cdots \leq \lambda_{m-2}} \prod_{i=1}^{m-2} q^{\lambda_{i}^{2}}\left[\begin{array}{c}
\lambda_{i+1} \\
\lambda_{i}
\end{array}\right]\left[\begin{array}{c}
n_{i}+n_{i+1} \\
n_{i}+\lambda_{i}
\end{array}\right],
\end{array}
$$

where $\lambda_{0}=0$ and $\lambda_{m-1}=n_{m}$. The last identity is clearly equivalent to that in Theorem 1.2.

In order to prove Theorem 1.3, we shall need the following relation:

$$
\begin{aligned}
& S\left(n_{1}, \ldots, n_{m} ; 0, q\right) \\
& \quad=S\left(n_{1}, \ldots, n_{m} ; m-1, q^{-1}\right) q^{n_{1} n_{2}+n_{2} n_{3}+\cdots+n_{m-1} n_{m}} .
\end{aligned}
$$

As $\left[\begin{array}{l}n \\ k\end{array}\right]_{q^{-1}}=\left[\begin{array}{l}n \\ k\end{array}\right] q^{k(k-n)}$, equation (2.7) can be verified by substituting $q$ by $q^{-1}$ and then replacing $k$ by $-k$ in the definition of $S\left(n_{1}, \ldots, n_{m} ; m-1, q\right)$. 
Proof of Theorem 1.3. We proceed by induction on $m \geq 1$. By the $q$ binomial theorem [6, Appendix, (II.3)], we have

$$
S\left(n_{1} ; 0, q\right)=\sum_{k=-n_{1}}^{n_{1}}(-1)^{k} q^{\left(\begin{array}{c}
k \\
2
\end{array}\right)}\left[\begin{array}{c}
2 n \\
n+k
\end{array}\right]=0 .
$$

In view of (2.5), it follows from (2.7) that

$$
S\left(n_{1}, n_{2} ; 0, q\right)=S\left(n_{1}, n_{2} ; 1, q^{-1}\right) q^{n_{1} n_{2}}=q^{n_{1} n_{2}} .
$$

So the theorem is valid for $m \leq 2$.

Now suppose that the expression $S\left(n_{1}, \ldots, n_{m-1} ; j, q\right)$ is a polynomial in $q$ with nonnegative integral coefficients for some $m \geq 3$ and $0 \leq j \leq$ $m-2$. Then by the recurrence formula $(2.3)$, so is $S\left(n_{1}, \ldots, n_{m} ; j, q\right)$ for $1 \leq j \leq m-1$. It remains to show that $S\left(n_{1}, \ldots, n_{m} ; 0, q\right)$ has the required property. Since the $q$-binomial coefficient $\left[\begin{array}{l}n \\ k\end{array}\right]$ is a polynomial in $q$ of degree $k(n-k)$ (see [2, p. 33]), it is easy to see from the definition that the degree of the polynomial $S\left(n_{1}, \ldots, n_{m} ; m-1, q\right)$ is less than or equal to

$$
n_{1} n_{2}+n_{2} n_{3}+\cdots+n_{m-1} n_{m} .
$$

It follows from $(2.7)$ that $S\left(n_{1}, \ldots, n_{m} ; 0, q\right)$ is also a polynomial in $q$ with nonnegative integral coefficients. This completes the inductive step of the proof.

REMARK. Though it is not necessary to check the $m=3$ case to validate our induction argument, we think it is convenient to include here the formulas for $m=3$. First, the $q$-Dixon identity (2.2) implies that

$$
S\left(n_{1}, n_{2}, n_{3} ; 1, q\right)=\left[\begin{array}{c}
n_{1}+n_{2}+n_{3} \\
n_{2}
\end{array}\right] .
$$

From (2.3) and (2.5) we derive

$$
S\left(n_{1}, n_{2}, n_{3} ; 2, q\right)=\sum_{l=0}^{n_{1}} q^{l^{2}}\left[\begin{array}{c}
n_{1} \\
l
\end{array}\right]\left[\begin{array}{c}
n_{2}+n_{3} \\
n_{2}-l
\end{array}\right] .
$$

Finally, applying (2.7) we get

$$
\begin{aligned}
S\left(n_{1}, n_{2}, n_{3} ; 0, q\right) & =S\left(n_{1}, n_{2}, n_{3} ; 2, q^{-1}\right) q^{n_{1} n_{2}+n_{2} n_{3}} \\
& =\sum_{l=0}^{n_{1}} q^{\left(n_{1}-l\right)\left(n_{2}-l\right)+n_{3} l}\left[\begin{array}{c}
n_{1} \\
l
\end{array}\right]\left[\begin{array}{c}
n_{2}+n_{3} \\
n_{2}-l
\end{array}\right] .
\end{aligned}
$$

3. Proof of Theorem 1.6. We will distinguish the cases where $r \geq 2$ is even or odd, and treat separately the values $r=2$ and $r=3$. 
- For $r=2$, apply (1.4) specialized with $m=1, a=q^{-(2 n+1)}, N=n-j$, $b_{1}=q^{-n}$ and $c_{1}=q^{-(n-j)}$. The left-hand side of (1.4) is then equal to

$$
\left[\begin{array}{c}
n+j \\
2 j
\end{array}\right]^{-2} q^{-2\left(\begin{array}{c}
n-j \\
2
\end{array}\right)+\left(\begin{array}{c}
n \\
2
\end{array}\right)} t_{n, j}^{(2)}(q) .
$$

Equating this with the right-hand side gives

$$
t_{n, j}^{(2)}(q)=\frac{(q)_{2 n}(q)_{j}^{2}}{(q)_{n}(q)_{2 j}(q)_{2 j-n}(q)_{n-j}^{2}} q^{2\left(\begin{array}{c}
n-j \\
2
\end{array}\right)-\left(\begin{array}{c}
n \\
2
\end{array}\right)},
$$

which shows that $\left[\begin{array}{c}2 j \\ j\end{array}\right]\left[\begin{array}{c}2 n \\ n\end{array}\right]^{-1} q^{\left(\begin{array}{c}n \\ 2\end{array}\right)} t_{n, j}^{(2)}(q) \in \mathbb{N}[q]$.

- For $r=3$, apply (1.4) specialized with $m=1, a=q^{-(2 n+1)}, N=n-j$ and $b_{1}=c_{1}=q^{-(n-j)}$. This yields in that case

$$
t_{n, j}^{(3)}(q)=\frac{(q)_{2 n}}{(q)_{3 j-n}(q)_{n-j}^{3}} q^{3\left(\begin{array}{c}
n-j \\
2
\end{array}\right)-2\left(\begin{array}{c}
n \\
2
\end{array}\right)},
$$

which shows that $\left[\begin{array}{c}2 j \\ j\end{array}\right]\left[\begin{array}{c}2 n \\ n\end{array}\right]^{-1} q^{2\left(\begin{array}{c}n \\ 2\end{array}\right)} t_{n, j}^{(3)}(q) \in \mathbb{N}[q]$.

- For $r=2 s \geq 4$, apply (1.4) with $m=s \geq 2, a=q^{-(2 n+1)}, N=n-j$, $b_{1}=q^{-n}$ and $c_{1}=b_{i}=c_{i}=q^{-(n-j)}$ for all $i \in\{2, \ldots, s\}$ to get

$$
\begin{aligned}
& q^{(2 s-1)\left(\begin{array}{c}
n \\
2
\end{array}\right)-2 s\left(\begin{array}{c}
n-j \\
2
\end{array}\right)} t_{n, j}^{(2 s)}(q) \\
&=\frac{(q)_{2 n}(q)_{j}}{(q)_{n}(q)_{2 j}(q)_{n-j}} \sum_{l_{1} \geq 0}\left[\begin{array}{c}
j \\
l_{1}
\end{array}\right]\left[\begin{array}{c}
n-l_{1} \\
j
\end{array}\right]\left[\begin{array}{l}
n-l_{1}+j \\
n-l_{1}-j
\end{array}\right] q^{\left(\begin{array}{c}
l_{1} \\
2
\end{array}\right)+2 j(s-1) l_{1}+(j+1-n) l_{1}} \\
& \times \sum_{l_{2} \geq 0}\left[\begin{array}{c}
2 j \\
l_{2}
\end{array}\right]\left[\begin{array}{c}
n-l_{1}-l_{2}+j \\
n-l_{1}-l_{2}-j
\end{array}\right]^{2} q^{\left(l_{2}\right)+2 j(s-2) l_{2}+(j+1-n) l_{2}} \times \cdots \\
& \times \sum_{l_{s-1} \geq 0}\left[\begin{array}{c}
2 j \\
l_{s-1}
\end{array}\right]\left[\begin{array}{l}
n-l_{1}-\cdots-l_{s-1}+j \\
n-l_{1}-\cdots-l_{s-1}-j
\end{array}\right]^{2} q^{\left(l_{s-1}\right)+2 j l_{s-1}+(j+1-n) l_{s-1}} \\
& \times\left[\begin{array}{c}
2 j \\
n-l_{1}-\cdots-l_{s-1}-j
\end{array}\right] q^{\left(l^{l_{1}+\cdots+l_{s-1}}\right)} .
\end{aligned}
$$

As the condition $l_{1}+\cdots+l_{s-1} \leq n-j$ holds in the last summation, we can see that for $s \geq 2,\left[\begin{array}{c}2 j \\ j\end{array}\right]\left[\begin{array}{c}2 n \\ n\end{array}\right]^{-1} q^{(2 s-1)\left(\begin{array}{c}n \\ 2\end{array}\right)} t_{n, j}^{(2 s)}(q) \in \mathbb{N}[q]$.

- For $r=2 s+1 \geq 5$, apply (1.4) with $m=s \geq 2, a=q^{-(2 n+1)}, N=n-j$ and $b_{i}=c_{i}=q^{-(n-\bar{j})}$ for all $i \in\{1, \ldots, s\}$ to get 


$$
\begin{aligned}
& q^{2 s\left(\begin{array}{c}
n \\
2
\end{array}\right)-(2 s+1)\left(\begin{array}{c}
n-j \\
2
\end{array}\right)} t_{n, j}^{(2 s+1)}(q) \\
&=\frac{(q)_{2 n}}{(q)_{2 j}(q)_{n-j}^{2}} \sum_{l_{1} \geq 0}\left[\begin{array}{l}
2 j \\
l_{1}
\end{array}\right]\left[\begin{array}{l}
n-l_{1}+j \\
n-l_{1}-j
\end{array}\right]^{2} q^{\left(\begin{array}{c}
l_{1} \\
2
\end{array}\right)+2 j(s-1) l_{1}+(j+1-n) l_{1}} \\
& \times \sum_{l_{2} \geq 0}\left[\begin{array}{c}
2 j \\
l_{2}
\end{array}\right]\left[\begin{array}{l}
n-l_{1}-l_{2}+j \\
n-l_{1}-l_{2}-j
\end{array}\right]^{2} q^{\left(\begin{array}{c}
l_{2} \\
2
\end{array}\right)+2 j(s-2) l_{2}+(j+1-n) l_{2}} \times \cdots \\
& \times \sum_{l_{s-1} \geq 0}\left[\begin{array}{c}
2 j \\
l_{s-1}
\end{array}\right]\left[\begin{array}{l}
n-l_{1}-\cdots-l_{s-1}+j \\
n-l_{1}-\cdots-l_{s-1}-j
\end{array}\right]^{2} q^{\left({ }^{l_{s-1}}\right)+2 j l_{s-1}+(j+1-n) l_{s-1}} \\
& \times\left[\begin{array}{l}
2 j \\
n-l_{1}-\cdots-l_{s-1}-j
\end{array}\right] q^{\left(\begin{array}{l}
l_{1}+\cdots+l_{s-1} \\
2
\end{array}\right) .}
\end{aligned}
$$

As the condition $l_{1}+\cdots+l_{s-1} \leq n-j$ holds in the last summation, we can see that for $s \geq 2,\left[\begin{array}{c}2 j \\ j\end{array}\right]\left[\begin{array}{c}2 n \\ n\end{array}\right]^{-1} q^{2 s\left(\begin{array}{c}n \\ 2\end{array}\right)} t_{n, j}^{(2 s+1)}(q) \in \mathbb{N}[q]$.

REMARK. In the special case $r=2$, our proof gives the following expression for the coefficients $c_{n}^{(2)}(q)$ :

$$
c_{n}^{(2)}(q)=\sum_{j=0}^{n}\left[\begin{array}{c}
2 j \\
n
\end{array}\right]\left[\begin{array}{l}
n \\
j
\end{array}\right]^{2} q^{2\left(\begin{array}{c}
n-j \\
2
\end{array}\right)} .
$$

These coefficients are $q$-analogues of the famous $c_{n}^{(2)}(1)$ involved in Apéry's proof of the irrationality of $\zeta(3)$ :

$$
c_{n}^{(2)}(1)=\sum_{j=0}^{n}\left(\begin{array}{c}
2 j \\
n
\end{array}\right)\left(\begin{array}{l}
n \\
j
\end{array}\right)^{2}=\sum_{j=0}^{n}\left(\begin{array}{l}
n \\
j
\end{array}\right)^{3} .
$$

As explained in [12], when $q=1$, one can derive the last expression from (3.1) in an elementary way (by two iterations of the Chu-Vandermonde formula). But our $q$-analogue (3.1) does not lead to a natural $q$-analogue of $(3.2)$.

4. Consequences of Theorems 1.2 and 1.3. Letting $q=1$ in Theorem 1.2 we obtain a direct generalization of Calkin's result (1.1).

TheOREM 4.1. For $m \geq 3$ and all positive integers $n_{1}, \ldots, n_{m}$,

$$
\begin{aligned}
& \sum_{k=-n_{1}}^{n_{1}}(-1)^{k} \prod_{i=1}^{m}\left(\begin{array}{c}
n_{i}+n_{i+1} \\
n_{i}+k
\end{array}\right) \\
& \quad=\left(\begin{array}{c}
n_{1}+n_{m} \\
n_{1}
\end{array}\right) \sum_{\lambda} \prod_{i=1}^{m-2}\left(\begin{array}{c}
\lambda_{i-1} \\
\lambda_{i}
\end{array}\right)\left(\begin{array}{c}
n_{i+1}+n_{i+2} \\
n_{i+1}-\lambda_{i}
\end{array}\right),
\end{aligned}
$$


where $n_{m+1}=\lambda_{0}=n_{1}$ and the sum is over all sequences $\lambda=\left(\lambda_{1}, \ldots, \lambda_{m-2}\right)$ of nonnegative integers such that $\lambda_{0} \geq \lambda_{1} \geq \cdots \geq \lambda_{m-2}$.

REMARK. For $m=1$ and 2, it is easy to see that the left-hand side of (4.1) is equal to 0 and $\left(\begin{array}{c}n_{1}+n_{2} \\ n_{1}\end{array}\right)$, respectively. Calkin's result follows from (4.1) by setting $n_{i}=n$ for all $i=1, \ldots, m$.

Letting $n_{1}=\cdots=n_{m}=n$ in Theorem 1.3, we obtain a complete $q$-analogue of Calkin's result.

Corollary 4.2. For all positive $m, n$ and $0 \leq j \leq m-1$,

$$
\left[\begin{array}{c}
2 n \\
n
\end{array}\right]^{-1} \sum_{k=-n}^{n}(-1)^{k} q^{j k^{2}+\left(\begin{array}{c}
k \\
2
\end{array}\right)}\left[\begin{array}{c}
2 n \\
n+k
\end{array}\right]^{m}
$$

is a polynomial in $q$ with nonnegative integral coefficients.

Letting $n_{2 i-1}=m$ and $n_{2 i}=n$ for $1 \leq i \leq r$ in Theorem 1.3, we obtain

Corollary 4.3. For all positive $m, n, r$ and $0 \leq j \leq 2 r-1$,

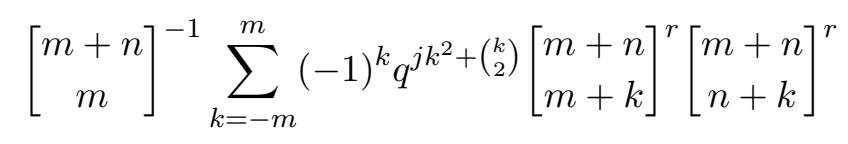

is a polynomial in $q$ with nonnegative integral coefficients. In particular,

$$
\sum_{k=-m}^{m}(-1)^{k}\left(\begin{array}{c}
m+n \\
m+k
\end{array}\right)^{r}\left(\begin{array}{c}
m+n \\
n+k
\end{array}\right)^{r}
$$

is divisible by $\left(\begin{array}{c}m+n \\ m\end{array}\right)$.

Letting $n_{3 i-2}=l, n_{3 i-1}=m$ and $n_{3 i}=n$ for $1 \leq i \leq r$ in Theorem 1.3, we obtain

Corollary 4.4. For all positive $l, m, n, r$ and $0 \leq j \leq 3 r-1$,

$$
\left[\begin{array}{c}
l+n \\
n
\end{array}\right]^{-1} \sum_{k=-l}^{l}(-1)^{k} q^{j k^{2}+\left(\begin{array}{c}
k \\
2
\end{array}\right)}\left[\begin{array}{l}
l+m \\
l+k
\end{array}\right]^{r}\left[\begin{array}{c}
m+n \\
m+k
\end{array}\right]^{r}\left[\begin{array}{l}
n+l \\
n+k
\end{array}\right]^{r}
$$

is a polynomial in $q$ with nonnegative integral coefficients. In particular,

$$
\sum_{k=-l}^{l}(-1)^{k}\left(\begin{array}{c}
l+m \\
l+k
\end{array}\right)^{r}\left(\begin{array}{c}
m+n \\
m+k
\end{array}\right)^{r}\left(\begin{array}{c}
n+l \\
n+k
\end{array}\right)^{r}
$$

is divisible by $\left(\begin{array}{c}l+m \\ l\end{array}\right),\left(\begin{array}{c}m+n \\ m\end{array}\right)$ and $\left(\begin{array}{c}n+l \\ n\end{array}\right)$.

Letting $m=2 r+s, n_{1}=n_{3}=\cdots=n_{2 r-1}=n+1$ and all the other $n_{i}$ be $n$ in Theorem 4.1, we get 
Corollary 4.5. For all positive $r, s$ and $n$,

$$
\sum_{k=-n}^{n}(-1)^{k}\left(\begin{array}{c}
2 n+1 \\
n+k+1
\end{array}\right)^{r}\left(\begin{array}{c}
2 n+1 \\
n+k
\end{array}\right)^{r}\left(\begin{array}{c}
2 n \\
n+k
\end{array}\right)^{s}
$$

is divisible by both $\left(\begin{array}{c}2 n \\ n\end{array}\right)$ and $\left(\begin{array}{c}2 n+1 \\ n\end{array}\right)$, and is therefore divisible by $(2 n+1)\left(\begin{array}{c}2 n \\ n\end{array}\right)$.

However, the following result is not a special case of Theorem 4.1.

Corollary 4.6. For all nonnegative $r$ and $s$ and positive $t$ and $n$,

$$
\sum_{k=-n}^{n}(-1)^{k}\left(\begin{array}{c}
2 n+1 \\
n+k+1
\end{array}\right)^{r}\left(\begin{array}{c}
2 n+1 \\
n+k
\end{array}\right)^{s}\left(\begin{array}{c}
2 n \\
n+k
\end{array}\right)^{t}
$$

is divisible by $\left(\begin{array}{c}2 n \\ n\end{array}\right)$.

Proof. We proceed by induction on $|r-s|$. The $r=s$ case is clear from Corollary 4.5. Suppose the statement is true for $|r-s| \leq m-1$. By Theorem 4.1, one sees that

$$
\begin{aligned}
& \text { (4.2) } \quad \sum_{k=-n}^{n}(-1)^{k}\left(\begin{array}{c}
2 n+2 \\
n+k+1
\end{array}\right)^{m}\left(\begin{array}{c}
2 n+1 \\
n+k+1
\end{array}\right)^{s}\left(\begin{array}{c}
2 n+1 \\
n+k
\end{array}\right)^{s}\left(\begin{array}{c}
2 n \\
n+k
\end{array}\right)^{t} \\
& =\frac{2 n+2}{2 n+1} \sum_{k=-n}^{n}(-1)^{k}\left(\begin{array}{c}
2 n+2 \\
n+k+1
\end{array}\right)^{m-1}\left(\begin{array}{c}
2 n+1 \\
n+k+1
\end{array}\right)^{s+1}\left(\begin{array}{c}
2 n+1 \\
n+k
\end{array}\right)^{s+1}\left(\begin{array}{c}
2 n \\
n+k
\end{array}\right)^{t-1},
\end{aligned}
$$

where $m, t \geq 1$, is divisible by

$$
\frac{2 n+2}{2 n+1}\left(\begin{array}{c}
2 n+1 \\
n
\end{array}\right)=2\left(\begin{array}{c}
2 n \\
n
\end{array}\right) .
$$

By the binomial theorem, we have

$$
\begin{aligned}
\left(\begin{array}{c}
2 n+2 \\
n+k+1
\end{array}\right)^{m} & =\left(\left(\begin{array}{c}
2 n+1 \\
n+k+1
\end{array}\right)+\left(\begin{array}{c}
2 n+1 \\
n+k
\end{array}\right)\right)^{m} \\
& =\sum_{i=0}^{m}\left(\begin{array}{c}
m \\
i
\end{array}\right)\left(\begin{array}{c}
2 n+1 \\
n+k+1
\end{array}\right)^{i}\left(\begin{array}{c}
2 n+1 \\
n+k
\end{array}\right)^{m-i} .
\end{aligned}
$$

Substituting (4.3) into the left-hand side of (4.2) and using the induction hypothesis and symmetry, we find that

$$
\begin{aligned}
& \sum_{k=-n}^{n}(-1)^{k}\left(\begin{array}{c}
2 n+1 \\
n+k+1
\end{array}\right)^{m+s}\left(\begin{array}{c}
2 n+1 \\
n+k
\end{array}\right)^{s}\left(\begin{array}{c}
2 n \\
n+k
\end{array}\right)^{t} \\
&+\sum_{k=-n}^{n}(-1)^{k}\left(\begin{array}{c}
2 n+1 \\
n+k+1
\end{array}\right)^{s}\left(\begin{array}{c}
2 n+1 \\
n+k
\end{array}\right)^{m+s}\left(\begin{array}{c}
2 n \\
n+k
\end{array}\right)^{t}
\end{aligned}
$$


is divisible by $2\left(\begin{array}{c}2 n \\ n\end{array}\right)$. However, replacing $k$ by $-k$, one sees that

$$
\begin{aligned}
& \sum_{k=-n}^{n}(-1)^{k}\left(\begin{array}{c}
2 n+1 \\
n+k+1
\end{array}\right)^{m+s}\left(\begin{array}{c}
2 n+1 \\
n+k
\end{array}\right)^{s}\left(\begin{array}{c}
2 n \\
n+k
\end{array}\right)^{t} \\
&=\sum_{k=-n}^{n}(-1)^{k}\left(\begin{array}{c}
2 n+1 \\
n+k+1
\end{array}\right)^{s}\left(\begin{array}{c}
2 n+1 \\
n+k
\end{array}\right)^{m+s}\left(\begin{array}{c}
2 n \\
n+k
\end{array}\right)^{t} .
\end{aligned}
$$

This proves that the statement is true for $|r-s|=m$.

It is clear that Theorems 1.3 and 4.1 can be restated in the following forms.

THEOREM 4.7. For all positive integers $n_{1}, \ldots, n_{m}$ and $0 \leq j \leq m-1$, the alternating sum

$$
(q)_{n_{1}} \prod_{i=1}^{m} \frac{(q)_{n_{i}+n_{i+1}}}{(q)_{2 n_{i}}} \sum_{k=-n_{1}}^{n_{1}}(-1)^{k} q^{j k^{2}+\left(\begin{array}{c}
k \\
2
\end{array}\right)} \prod_{i=1}^{m}\left[\begin{array}{c}
2 n_{i} \\
n_{i}+k
\end{array}\right],
$$

where $n_{m+1}=0$, is a polynomial in $q$ with nonnegative integral coefficients.

THEOREM 4.8. For all positive integers $n_{1}, \ldots, n_{m}$, we have

$$
n_{1} ! \prod_{i=1}^{m} \frac{\left(n_{i}+n_{i+1}\right) !}{\left(2 n_{i}\right) !} \sum_{k=-n_{1}}^{n_{1}}(-1)^{k} \prod_{i=1}^{m}\left(\begin{array}{c}
2 n_{i} \\
n_{i}+k
\end{array}\right) \in \mathbb{N},
$$

where $n_{m+1}=0$.

It is easy to see that, for all positive integers $m$ and $n$, the expression $(2 m) !(2 n) ! /(m+n) ! m ! n !$ is an integer by considering the power of a prime dividing a factorial. Letting $n_{1}=\cdots=n_{r}=m$ and $n_{r+1}=\cdots=n_{r+s}=n$ in Theorem 4.8, we obtain

Corollary 4.9. For all positive $m, n, r$ and $s$,

$$
\sum_{k=-m}^{m}(-1)^{k}\left(\begin{array}{c}
2 m \\
m+k
\end{array}\right)^{r}\left(\begin{array}{c}
2 n \\
n+k
\end{array}\right)^{s}
$$

is divisible by $(2 m) !(2 n) ! /(m+n) ! m ! n !$.

In particular, we find that

$$
\sum_{k=-n}^{n}(-1)^{k}\left(\begin{array}{c}
4 n \\
2 n+k
\end{array}\right)^{r}\left(\begin{array}{c}
2 n \\
n+k
\end{array}\right)^{s}
$$

is divisible by $\left(\begin{array}{c}4 n \\ n\end{array}\right)$, and

$$
\sum_{k=-n}^{n}(-1)^{k}\left(\begin{array}{c}
6 n \\
3 n+k
\end{array}\right)^{r}\left(\begin{array}{c}
2 n \\
n+k
\end{array}\right)^{s}
$$

is divisible by $(6 n) !(2 n) ! /(4 n) !(3 n) ! n !$. 
From Theorem 4.8 it is easy to see that

$$
n_{1} ! \prod_{i=1}^{m} \frac{\left(n_{i}+n_{i+1}\right) !}{\left(2 n_{i}\right) !} \sum_{k=-n_{1}}^{n_{1}}(-1)^{k} \prod_{i=1}^{m}\left(\begin{array}{c}
2 n_{i} \\
n_{i}+k
\end{array}\right)^{r_{i}}
$$

where $n_{m+1}=0$, is a nonnegative integer for all $r_{1}, \ldots, r_{m} \geq 1$. For $m=3$, letting $\left(n_{1}, n_{2}, n_{3}\right)$ be $(n, 3 n, 2 n),(2 n, n, 3 n)$, or $(2 n, n, 4 n)$, we obtain the following two corollaries.

Corollary 4.10. For all positive $r, s, t$ and $n$,

$$
\sum_{k=-n}^{n}(-1)^{k}\left(\begin{array}{c}
6 n \\
3 n+k
\end{array}\right)^{r}\left(\begin{array}{c}
4 n \\
2 n+k
\end{array}\right)^{s}\left(\begin{array}{c}
2 n \\
n+k
\end{array}\right)^{t}
$$

is divisible by both $\left(\begin{array}{c}6 n \\ n\end{array}\right)$ and $\left(\begin{array}{c}6 n \\ 3 n\end{array}\right)$.

Corollary 4.11. For all positive $r, s, t$ and $n$,

$$
\sum_{k=-n}^{n}(-1)^{k}\left(\begin{array}{c}
8 n \\
4 n+k
\end{array}\right)^{r}\left(\begin{array}{c}
4 n \\
2 n+k
\end{array}\right)^{s}\left(\begin{array}{c}
2 n \\
n+k
\end{array}\right)^{t}
$$

is divisible by $\left(\begin{array}{l}8 n \\ 3 n\end{array}\right)$.

5. Some open problems. Based on computer experiments, we would like to present four interesting conjectures. The first two are refinements of Corollaries 4.10 and 4.11 respectively.

Conjecture 5.1. For all positive $r, s, t$ and $n$,

$$
\sum_{k=-n}^{n}(-1)^{k}\left(\begin{array}{c}
6 n \\
3 n+k
\end{array}\right)^{r}\left(\begin{array}{c}
4 n \\
2 n+k
\end{array}\right)^{s}\left(\begin{array}{c}
2 n \\
n+k
\end{array}\right)^{t}
$$

is divisible by both $2\left(\begin{array}{c}6 n \\ n\end{array}\right)$ and $6\left(\begin{array}{c}6 n \\ 3 n\end{array}\right)$.

Conjecture 5.2. For all positive $r, s$, $t$ and $n$ with $(r, s, t) \neq(1,1,1)$,

$$
\sum_{k=-n}^{n}(-1)^{k}\left(\begin{array}{c}
8 n \\
4 n+k
\end{array}\right)^{r}\left(\begin{array}{c}
4 n \\
2 n+k
\end{array}\right)^{s}\left(\begin{array}{c}
2 n \\
n+k
\end{array}\right)^{t}
$$

is divisible by $2\left(\begin{array}{l}8 n \\ 3 n\end{array}\right)$.

Conjectures 5.1 and 5.2 are true for $r+s+t \leq 10$ and $n \leq 100$.

Let $\operatorname{gcd}\left(a_{1}, a_{2}, \ldots\right)$ denote the greatest common divisor of integers $a_{1}, a_{2}, \ldots$

Conjecture 5.3. For all positive $m$ and $n$, we have

$$
\operatorname{gcd}\left(\sum_{k=-n}^{n}(-1)^{k}\left(\begin{array}{c}
2 n \\
n+k
\end{array}\right)^{r}, r=m, m+1, \ldots\right)=\left(\begin{array}{c}
2 n \\
n
\end{array}\right) \text {. }
$$


Let $d \geq 2$ be a fixed integer. Every nonnegative integer $n$ can be uniquely written as

$$
n=\sum_{i \geq 0} a_{i} d^{i}
$$

where $0 \leq a_{i} \leq d-1$ for all $i$ and only finitely many numbers of $b_{i}$ are nonzero, denoted by $n=\left[\cdots a_{1} a_{0}\right]_{d}$, in which the first 0 's are omitted. Let $n=\left[a_{1} \cdots a_{r}\right]_{3}=\left[b_{1} \cdots b_{s}\right]_{7}=\left[c_{1} \cdots c_{t}\right]_{13}$. We now define three statistics $\alpha(n), \beta(n)$ and $\gamma(n)$ as follows:

- Let $\alpha(n)$ be the number of disconnected 2's in the sequence $a_{1} \cdots a_{r}$. Here two nonzero digits $a_{i}$ and $a_{j}$ are said to be disconnected if there is at least one 0 between $a_{i}$ and $a_{j}$.

- Let $\beta(n)$ be the number of 1 's in $b_{1} \cdots b_{s}$ which are not immediately followed by a 4,5 , or 6 .

- Let $\gamma(n)$ be the number of 1's in $c_{1} \cdots c_{t}$ which are immediately followed by one of $7, \ldots, 12$, or immediately followed by a number of 6 's and then by one of $7, \ldots, 12$.

For instance, $[20212]_{3}=185$ and so $\alpha(185)=2 ;[10142]_{7}=2480$, and so $\beta(2480)=1 ;[1667]_{13}=3296$ and so $\gamma(3296)=1$. The first $n$ such that $\alpha(n)=4$ is $[2020202]_{3}=1640$; the first $n$ such that $\beta(n)=4$ is $[1111]_{7}=$ 400; while the first $n$ such that $\gamma(n)=4$ is $[17171717]_{13}=97110800$.

We end this paper with the following conjecture.

Conjecture 5.4. For every positive integer $n$,

$$
\begin{gathered}
\operatorname{gcd}\left(\sum_{k=-n}^{n}(-1)^{k}\left(\begin{array}{c}
2 n \\
n+k
\end{array}\right)^{3 r}, r=1,2, \ldots\right)=\left(\begin{array}{c}
2 n \\
n
\end{array}\right) 3^{\alpha(n)}, \\
\operatorname{gcd}\left(\sum_{k=-n}^{n}(-1)^{k}\left(\begin{array}{c}
2 n \\
n+k
\end{array}\right)^{3 r+1}, r=1,2, \ldots\right)=\left(\begin{array}{c}
2 n \\
n
\end{array}\right) 7^{\beta(n)} 13^{\gamma(n)}, \\
\operatorname{gcd}\left(\sum_{k=-n}^{n}(-1)^{k}\left(\begin{array}{c}
2 n \\
n+k
\end{array}\right)^{3 r+2}, r=1,2, \ldots\right)=\left(\begin{array}{c}
2 n \\
n
\end{array}\right) .
\end{gathered}
$$

Acknowledgments. This work was partially done during the first author's visit to Institut Camille Jordan, Université Claude Bernard (Lyon 1), and was supported by a French postdoctoral fellowship. We thank Christian Krattenthaler for conveying us his feeling that Theorem 1.2 should follow from Andrews' formula [1, Theorem 4]. We also thank Wadim Zudilin for useful conversations during his visit in Lyon. Finally, the first author is grateful to Hong-Xing Ding for helpful comments on Conjecture 5.2. 


\section{References}

[1] G. E. Andrews, Problems and prospects for basic hypergeometric functions, in: Theory and Application of Special Functions, R. A. Askey (ed.), Math. Res. Center Univ. of Wisconsin Publ. No. 35, Academic Press, New York, 1975, 191-224.

[2] - , The Theory of Partitions, Cambridge Univ. Press, Cambridge, 1998.

[3] N. G. de Bruijn, Asymptotic Methods in Analysis, Dover, New York, 1981.

[4] N. J. Calkin, Factors of sums of powers of binomial coefficients, Acta Arith. 86 (1998), 17-26.

[5] L. Carlitz, Some inverse relations, Duke Math. J. 40 (1973), 893-901.

[6] G. Gasper and M. Rahman, Basic Hypergeometric Series, 2nd ed., Encyclopedia Math. Appl. 96, Cambridge Univ. Press, Cambridge, 2004.

[7] V. J. W. Guo, F. Jouhet and J. Zeng, New finite Rogers-Ramanujan identities, preprint, 2006, arXiv: math.CO/0604407.

[8] V. J. W. Guo and J. Zeng, A combinatorial proof of a symmetric q-Pfaff-Saalschütz identity, Electron. J. Combin. 12 (2005), \#N2.

[9] -, 一, A short proof of the q-Dixon identity, Discrete Math. 296 (2005), 259-261.

[10] C. Krattenthaler, A new matrix inverse, Proc. Amer. Math. Soc. 124 (1996), 47-59.

[11] A. L. Schmidt, Generalized q-Legendre polynomials, J. Comput. Appl. Math. 49 (1993), 243-249.

[12] V. Strehl, Binomial identities - combinatorial and algorithmical aspects, Discrete Math. 136 (1994), 309-346.

[13] D. Zeilberger, A q-Foata proof of the q-Saalschütz identity, European J. Combin. 8 (1987), 461-463.

[14] W. Zudilin, On a combinatorial problem of Asmus Schmidt, Electron. J. Combin. 11 (2004), \#R22.

Department of Mathematics

East China Normal University

Shanghai 200062, People's Republic of China

E-mail: jwguo@math.ecnu.edu.cn

http://math.ecnu.edu.cn/ jwguo
Institut Camille Jordan

Université Lyon 1

Bâtiment du Doyen Jean Braconnier

43, blvd. du 11 novembre 1918

F-69622 Villeurbanne Cedex, France

E-mail: jouhet@math.univ-lyon1.fr http://math.univ-lyon1.fr/ jouhet zeng@math.univ-lyon1.fr

http://math.univ-lyon1.fr/ zeng 